Both vibration and dissociation rates are sensitive to the presence of impurities. Addition of 2 per cent water vapour reduced the dissociation time to 100 usec. for a shock with equilibrium temperature $2,445^{\circ} \mathrm{K}$. The vibrational relaxation time was measured as only $23 \mu \mathrm{sec}$. at $2,270^{\circ}$, and was too short to record at the higher temperatures for which results were obtained for pure carbon dioxide.

Carbon dioxide possesses three vibrational modes ; the symmetrical mode $v_{1}$ has $a$ frequency of 1,388 cm..$^{-1}$, the transverse mode $\nu_{2}$ one of $667 \mathrm{~cm}^{-1}$ and the asymmetric mode $\nu_{3}$ one of $2,349 \mathrm{~cm}^{-1}$. Ultrasonic dispersion is dominated by relaxation of the lowest frequency mode $\nu_{2}$ and shows a relaxation time which at high temperature would be much too short to observe under our conditions. $v_{2}$ makes a smaller contribution to the specific heat at low temperature and does not show a well-defined dispersion frequency ; it is usually assumed that inter-conversion of energy from one mode to another on collision would lead to the two frequencies having the same relaxation time, although there is at least one report ${ }^{4}$ of a shelf on the sound dispersion curve which might be identified with separate relaxation of $\nu_{1}$. The asymmetric mode $v_{3}$ has too high a frequency to make an appreciable contribution to the specific heat in the temperature range of ultra-sonic studies. It has seemed uncertain whether a separate relaxation time should exist for this high-frequency mode or whether interconversion of energy between the three vibrational forms should lead to a single relaxation time. For sulphur dioxide, there is evidence for two separate relaxation times ${ }^{5}$. The present results appear to indicate that carbon dioxide has a separate relaxation time for the asymmetric mode which is of the same order as the vibrational relaxation times of nitrogen and carbon monoxide, which have comparable vibrational frequencies $\left(2,360 \mathrm{~cm}^{-1}\right.$ and $2,170 \mathrm{~cm} .^{-1}$ respectively). This separate relaxation of $v_{3}$ seems reasonable, since it is difficult to visualize an efficient process of transfer of vibrational energy between the asymmetric and valence modes.

The relatively long time for the dissociation of carbon dioxide and the influence of water on this is of interest for the time to attain equilibrium following combustion. There is much evidence for delayed burning of carbon monoxide and for the action of water vapour as catalyst.

\section{R. HuRLE}

A. G. Gaydon

Chemical Engineering Department,

Imperial College of Science and Technology,

$$
\text { London, S.W.7. }
$$

Nov. 2 .

${ }^{1}$ Clouston, J. G., Gaydon, A. G., and Glass, I. I., Proc. Roy. Soc., A.,

2 248, 429 (1958). A., 252, 143 (1959)

3 Griffith, W. C., and Kenny, A., Princeton Tech. Report II-23 (March,

$1957)$.

- Pielemier, W. H., Saxton, H. L., and Telfair, D., J. Chem. Phys., 8, $106(1940)$

- Lambert, J. D., and Salter, R., Proc. Roy. Soc., A, 243, 78 (1958)

\section{Phase Transitions in Interlamellar Films}

A Prevrous communication ${ }^{1}$ has described the interlamellar sorption of straight chain amines by graphic acid. Attention was then directed to a break in the curve of spacing against chain-length, occurring between 9 and $10 \mathrm{C}$-atoms per molecule.

This we now believe to be due to the change of the sorbed film from the liquid to the solid state. If this assumption is accepted, it follows that the sorbed films solidify more readily than the pure substances, for which the liquid-solid transition occurs between 11 and 12 C-atoms.

Evidence for the assumption is provided by observation, on the X-ray diagram, of a diffraction in the region of $4 \cdot 3 \mathrm{~A}$. This consists of a broad band on the diagram of the complexes with the lower amines, but it sharpens to a sharp line concurrently with the spacing shift. This band or line is presumably due to the spacing between neighbouring chains.

Further evidence is provided by $\mathrm{X}$-ray diagrams of sorption complexes with mixtures of two amines, that is, hexylamine and decylamine. These give intermediate spacings which, when plotted against the proportion of higher amines in the mixture (Fig. 1) give a straight line. The point for pure

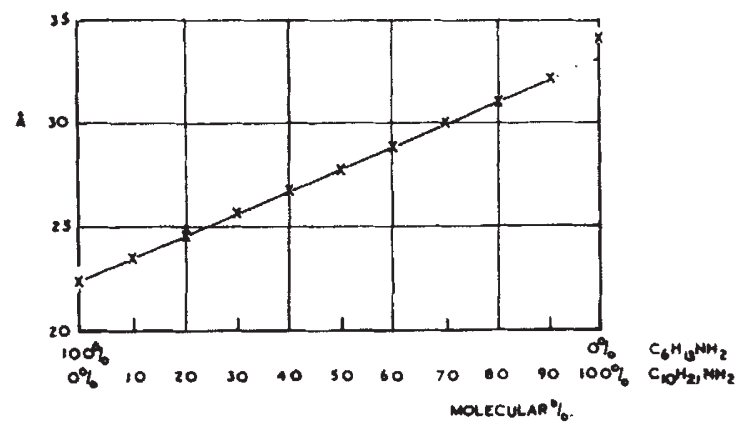

Fig. 1. Interlayer spacings of graphitic acid complexes with mixtures of hexylamine and decylamine, plotted against proportion

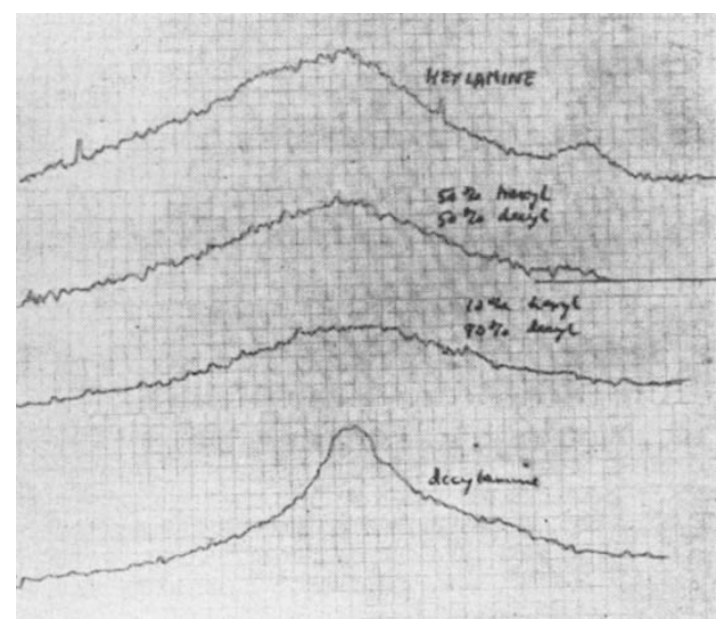

Fig. 2. Microphotometer curves of the diffraction maximum in the region of $4 \cdot 3 \mathrm{~A}$. for certain of the above mixtures in Fig. 1

decylamine, however, lies off the sharper line. Here again, the nature of the diffraction at $4 \cdot 3 \mathrm{~A}$. changes concurrently with the break in the graph (Fig. 2). We have made several similar observations.

Detailed observation suggests that the liquidsolid change in these sorbed films is not sharp, but that the $4 \cdot 3 \mathrm{~A}$. line sharpens over a range of values following the break.

Carnegie Laboratory of Physics,

D. M. C. MacEwaN

F. Aragón de la Cruz

Queen's College,

Dundee.

Aug. 13. ${ }^{3}$ Aragón, F., Cano Ruiz, J., and MacEwan, D. M. C., Nature, 183, 740 